\title{
Ameliorative Effect of Sodium Selenite on Silver Nanoparticles-Induced Myocardiocyte Structural Alterations in Rats
}

This article was published in the following Dove Press journal: International Journal of Nanomedicine

\author{
Wanrui Ma ${ }^{1,2}$ \\ Shan $\mathrm{He}^{3}$ \\ Yanping $\mathrm{Xu}^{4}$ \\ Guoxue $\mathrm{Qi}^{3}$ \\ Huiyan $\mathrm{Ma}^{3}$ \\ John J Bang $\mathbb{D}^{5}$ \\ $P$ Andy $\mathrm{Li}^{2}$ \\ 'Department of General Medicine, \\ General Hospital of Ningxia Medical \\ University, Yinchuan, Ningxia, People's \\ Republic of China; ${ }^{2}$ Department of \\ Pharmaceutical Sciences, \\ Biomanufacturing Research Institute and \\ Technological Enterprise (BRITE), North \\ Carolina Central University, Durham, \\ NC, USA; ${ }^{3}$ Ningxia Medical University, \\ Yinchuan, Ningxia, People's Republic of \\ China; ${ }^{4}$ Unit of Echocardiography, \\ Division of Functional Examination in \\ Heart Center, General Hospital of \\ Ningxia Medical University, Yinchuan, \\ Ningxia, People's Republic of China; \\ ${ }^{5}$ Department of Environmental, Earth and \\ Geospatial Sciences, North Carolina \\ Central University, Durham, NC, USA
}

Background: The application of silver nanoparticles (AgNPs) is growing exponentially, and its potential damage to the cardiac remains to be elucidated. The purpose of this study was to investigate the ameliorative effect of sodium selenite on silver nanoparticles-induced myocardiocyte structural alterations in rats.

Materials and Methods: Forty male Sprague-Dawley (SD) rats were randomly divided into four groups: control group, AgNPs group, Se control group, and AgNPs + Se group. SD rats were administered AgNPs through a single intratracheal instillation, and sodium selenite was given by intraperitoneal injection for seven days. Cardiac function was determined by echocardiography and hemodynamic, ultrastructural changes by transmission electron microscopy examination. Mitochondrial fission and autophagy markers were measured by Western blotting.

Results: AgNPs caused a significant decrease in cardiac contraction, diastolic dysfunction, fragmentation, and lysis of the myofibrils, the formation of stenosis in the capillary, damaging the mitochondria membrane and cristae. AgNPs significantly increased mitochondrial fission markers dynamin-related protein 1 (Drp1), phospho-Drp1 (p-Drp1), and mitochondrial fission protein 1 (Fis1), as well as autophagy marker LC3 II/I $(P<0.05)$. Treatment with sodium selenite is capable of protecting cardiac function from AgNPs toxicity through attenuating ultrastructural alterations, stabilizing mitochondrial dynamic balance and blocking mitochondrial autophagy.

Conclusion: We conclude that the protection of sodium selenite against silver nanoparticlesinduced myocardiocyte structural alterations is associated with stabilizing mitochondrial dynamic balance and mitophagy.

Keywords: heart, mitochondria, mitochondrial fission, myocardiocyte, myofibril, sarcomere, silver nanoparticles, sodium selenite

\section{Introduction}

Silver nanoparticles (AgNPs), a potent broad-spectrum antibacterial nanomaterial, has widely been used for daily necessities and biomedical purposes. ${ }^{1}$ ith the increased application of AgNPs, a large number of AgNPs products have been produced every year. It is estimated that the United States alone produces about 2.8 to 20 tons per year. ${ }^{2}$ Approximately $14 \%$ of the AgNPs-containing products can release these nanoparticles by manipulation. ${ }^{3,4}$ This represents a potential source of environmental and biological exposure with serious concerns for human health and our ecosystems. Inhalation has been considered the most common route of exposure for AgNPs. ${ }^{5,6}$

\section{Correspondence: $\mathrm{P}$ Andy $\mathrm{L}$}

of Pharmaceutical Scienc

Biomanufacturing Research Institute and Technological Enterprise (BRITE), North Carolina Central University, I80 I

Fayetteville Street, Durham, NC 27707 USA

Tel + I 9195306872

$\mathrm{Fax}+19195306600$

Email pli@nccu.edu
International Journal of Nanomedicine 2020:15 828I-8292

DovePress in $\mathbf{D}$ 
AgNPs can pass through the lung barriers into the blood and disperse throughout the body into organs such as the heart, liver, spleen, kidney, and brain. ${ }^{7,8}$ The accumulation of AgNPs in the heart has been observed, albeit at a low concentration. $^{8}$ Therefore, the cardiac risk and toxicity mechanism of AgNPs have not attracted sufficient attention. In fact, cardiac toxicity of AgNPs has been demonstrated in some studies, as reflected by myocardiocyte deformity, rhabdomyolysis, increase in vasoconstriction, aggravation of cardiac ischemia and reperfusion injury, and decrease of cardiac contraction. $^{9-12}$ The mechanisms of its cardiac toxicity are not fully understood but may involve several different mechanisms, such as cytotoxicity and genotoxicity, inflammatory responses, oxidative stress, and down-regulation of certain genes. ${ }^{13-16}$ The present study focuses on studying the mitochondrial function, morphology, and fission related proteins in the rats exposed to AgNPs inhalation.

Mitochondria, the major sources of energy, are essential and extremely important for the heart because it needs huge amounts of energy to keep contraction. Studies have shown that AgNPs-induced mitochondrial toxicity is mainly mediated by oxidative stress, mitochondrial depolarization, and inhibition of key mitochondrial enzymes. ${ }^{17}$ However, mitochondria dynamic balance, the equilibrium of mitochondrial fission and fusion, is essential and important for the health of the organism. Mitochondrial fusion enables the exchange of matrix components, as well as damaged mtDNA. ${ }^{18}$ On the contrary, fission leads to activation of apoptosis and autophagy, ${ }^{19}$ allowing more mitochondria to distribute further or package degenerated protein for lysis and recycling. $^{20,21}$ Fission is mainly regulated by Drp1 and Fis1 in mammals. ${ }^{22}$ Our previous research showed that AgNPs exposure induced mitochondrial stress in a neuronal cell line and damage in the lung tissue. ${ }^{23,24}$ So far, the effect of AgNPs on mitochondrial dynamic balance in myocardiocytes has not been reported. In particular, there is little evidence for the transport of AgNPs to the mitochondria in the myocardiocytes.

Finding amelioration methods to protect against AgNPs-induced cardiac toxicity is equally important and imminent. Selenium, an essential micronutrient, is well-known for its detoxification effects in heavy metal exposure. ${ }^{25}$ Selenium is a central component of various antioxidant enzyme catalytic sites, and it can restore the activity of antioxidant enzymes, such as glutathione peroxidases and thioredoxin reductase (TrxR). ${ }^{26-28}$ A study indicated that exposure to AgNPs caused inhibition of selenoprotein TrxR synthesis. ${ }^{29}$ Further, AgNPs induced the changes in the proteome, which may be related to the antagonistic effect of silver on the status of selenium. ${ }^{30}$ Our previous study showed that selenium can block AgNPs-caused caspase-3 activation, prevent mitochondrial dysfunction and pulmonary structural damage. ${ }^{23,24}$ However, it is not clear whether sodium selenite is capable of preventing damage caused by AgNPs. Our aim in this study was to examine the mechanism by which sodium selenite protects against the cardiac damage induced by AgNPs exposure.

\section{Materials and Methods Materials}

The silver nanoparticles powder was obtained from US Research Nanomaterials, Inc $(20 \mathrm{~nm}$, Product Number: US1038), which is the same as we have used in a recent study. $^{24}$ AgNPs were suspended in $0.9 \% \mathrm{NaCl}$ and reserved as stock solution (concentration $1 \mathrm{mg} / \mathrm{mL}$ ). To prevent the aggregation, AgNPs stock suspension was sonicated in an ice bath at room temperature for $30 \mathrm{~min}$ before intratracheal instillation (IT) to the experimental rats. Selenium was purchased from Sigma Company and dissolved in $0.9 \% \mathrm{NaCl}$ before use.

\section{Animals}

Forty male Sprague-Dawley rats, obtained from the Animal Center of Ningxia Medical University, China, were used in the present study. The animals were 6-8 weeks of age and weighed 180-220 grams. They were maintained in plastic cages under controlled room temperatures $\left(22 \pm 4^{\circ} \mathrm{C}\right)$ and humidity $(30-70 \%)$, and subjected to a 12 hours light/dark cycle. Standard rodent chow and sterile water were provided ad libidum. After a week of acclimatization, the rats were randomly divided into four groups: 1) Control group, $0.9 \%$ saline IT and $0.9 \%$ saline IP; 2) AgNPs group, AgNPs IT and 0.9\% saline IP; 3) Se control group, $0.9 \%$ saline IT and selenite IP; and 4) AgNPs + Se group, AgNPs IT and selenite IP). Each group consists of ten rats. Intratracheal instillation (IT) was conducted only once with a volume of 200 microliters of AgNPs solution or $0.9 \%$ saline. Sodium selenite $(0.2 \mathrm{mg} / \mathrm{Kg}$ body weight in $1 \mathrm{~mL})$ or $0.9 \%$ saline was intraperitoneally injected daily for 7 days started concurrently with the AgNPs intratracheal instillation. Upon predetermined endpoint, the rats were sacrificed under intraperitoneal anesthesia with $10 \%$ chloral hydrate $(0.3-$ $0.4 \mathrm{~mL} / 100 \mathrm{~g}$ body weight). 
The animal experiments and all the procedures were approved by the Institutional Review Board of the General Hospital of Ningxia Medical University (approval numbers 2016-089) and performed in strict accordance with the Guide for Laboratory Animal Care and Use.

\section{Weights of the Heart and Body}

Body weight was recorded twice a week and at the time of euthanasia. The heart was removed from the animal and immediately washed three times in ice-cold saline. Wet weights of the heart were measured after dried with a regular filter. The ratio of heart weight over the body weight in each rat was calculated and presented.

\section{Echocardiography Examination}

On the 21st day after intratracheal instillation, six rats from each group were randomly selected for transthoracic echocardiography using the Vivid 7 Dimension system (GE Healthcare Ultrasound, Horten, Norway) with a 12 $\mathrm{MHz}$ transducer. The rats were laid on their back under the maintenance of $1-1.5 \%$ isoflurane anesthesia, a small dose that did not affect the level of heart rate, blood pressure, and the state of respiration in rats at any stage. The parameters measured were the following: pulmonary artery internal diameter (PA), interventricular septum thickness (IVS), the left ventricular internal dimension at the end of diastole (LVIDd), left ventricular internal dimension at the end of systole (LVIDs), heart rate (HR). The left ventricular fractional shortening (LVFS), ejection fraction (EF), cardiac output (CO), stroke volume (SV), the Left ventricular volume at the end of diastole (EDV) and the Left ventricular volume at the end of systole (ESV) were calculated according to traditional formulas(as shown in sup plementary material-experimentary section). All parameters were measured 3-4 times, and the images were captured by the same operator who was specialized in small animal echocardiography and blinded to the study groups.

\section{Hemodynamic Examination}

After echocardiography, the cardiac function was assessed by measuring hemodynamic parameters through the insertion of a cardiovascular catheter to the left ventricular of the heart in the experimental rats. The data were acquired using a computer-controlled BL-420S physiological signal acquisition system (Chengdu Taimeng Software Co. Ltd, China). The connected polyethylene catheter and pressure transducer were filled with $500 \mathrm{U} / \mathrm{mL}$ of heparin saline (avoiding air bubbles as possible). The rats were anesthetized with an intraperitoneal injection of $20 \%$ urethane $(6.5 \mathrm{~mL} / \mathrm{Kg}$ body weight) and fixed on the operating table, connected to the standard II lead electrocardiogram, and the heart rate was recorded. The trachea was surgically exposed, then the right carotid artery was isolated. The distal end of the carotid artery was ligated and the proximal end was clipped using an artery clip. An oblique incision was then made with microscope scissors. The catheter was introduced into the right common carotid and reached to the left ventricle, where significant increases of pressure and waveform were observed on the computer screen. After all the steps were completed, the hemodynamic parameters were recorded once they reached a steady-state for about 10 minutes. The record of hemodynamic responses in each rat was continued for 30 minutes. The parameters were measured as follows: heart rate (HR), left ventricular systolic pressure (LVSP), left ventricular end-diastolic pressure (LVEDP), maximal rate of left ventricular pressure rise $\left(\mathrm{LVdP} / \mathrm{dt}_{\max }\right)$, and maximal rate of pressure decrease during relaxation $\left(\mathrm{LVdP} / \mathrm{dt}_{\min }\right)$.

\section{Transmission Electron Microscopy Examination}

Parts of cardiac tissue was obtained immediately after the animals were sacrificed. The heart was cut into small pieces $\left(1 \mathrm{~mm}^{3}\right)$, fixed in $2 \%$ glutaraldehyde, rinsed in $0.1 \mathrm{M}$ dimethyl sodium arsenate, and followed by postfixation in $4 \%$ osmic acid, washing with $0.1 \mathrm{M}$ dimethyl sodium arsenate, and dehydration in ascending concentrations of alcohol. All the above processes were carried out at $4{ }^{\circ} \mathrm{C}$. The tissues were permeated in propylene oxide and then embedded in epoxy resin for 48 hours under $60^{\circ} \mathrm{C}$. The ultra-thin sections were cut and stained with $2 \%$ uranyl acetate and lead citrate. All images were captured by a TEM (Hitachi H-7650, Tokyo, Japan).

\section{Western Blot}

The total proteins and mitochondrial proteins were extracted from heart tissues using the total protein extraction kit (KGP2100) and mitochondrial protein extraction kit (KGP8100) from KeyGEN BioTECH. The extraction processes were conducted in an ice-water bath according to the instructions of the kits. The protein concentration was determined using the BCA Protein Assay Kit (KeyGEN BioTECH, KGP8100). Equal amounts of protein $(20 \mu \mathrm{g})$ from each animal subjected to heat denaturation were loaded for electrophoresis and transferred onto nitrocellulose membranes. The membrane was blocked with $10 \%$ skim milk at 
room temperature and incubated overnight at $4^{\circ} \mathrm{C}$ with the following primary antibodies: Drp1 (1:1000, ab184247, Abcam), phospho-Drp1 (Ser616 1:1000, 3455, Cell Signaling), Fis1 (1:1000, GTX111010, GeneTex), LC3B (1:2000, ab192890, Abcam) and VDAC (1:1000, GTX114187, GeneTex). The membranes were washed and incubated with horseradish peroxidase-labeled secondary antibodies for 1 hour at room temperature, followed by imaging using the Pierce ECL Western Blotting Substrate. The VDAC bands served as a mitochondrial and GAPDH as cytosolic internal controls. The ratios of targeted protein bands to loading controls were measured and presented as relative target band intensity.

\section{Statistical Analysis}

All data are expressed as mean \pm standard deviation. SPSS 18.0 software and Graph Pad Prism 5.0 were used for the data analysis. Differences between groups in mean values with normal distribution were compared by two-way ANOVA followed by Bonferroni Test. $P<0.05$ was considered to indicate a statistically significant difference.

\section{Results}

\section{Body Weight and Heart Coefficient}

Body weight and heart weight of each rat were compared among the four groups. As shown in Figure 1, the bodyweight of all four groups of rats increased over time. On the 21st day after intratracheal instillation, the mean body weights were $(320.50 \pm 24.73) \mathrm{g}$, (328.67 \pm 18.82$) \mathrm{g}$, (343.17 $\pm 22.59) \mathrm{g}$ and $(329.50 \pm 25.56) \mathrm{g}$ in control, AgNPs-treated, Se-treated, and AgNPs-Se-cotreated groups, respectively. The heart coefficient, which is calculated by dividing heart weight with body weight in the same animal, was $(2.80-$ $3.29) \times 10^{-3}$ in all animals with no significant difference between the groups $(\mathrm{F}=0.228, \mathrm{p}=0.876)$. The data indicate that AgNPs did not induce significant changes in body weight and heart coefficients.

\section{Assessment of Cardiac Function by Echocardiography and Invasive Hemodynamics}

Cardiac parameters, including PA, IVS, LVIDd, LVIDs, SV, LVFS, EF, EDV, and ESV, which were obtained from echocardiography, were presented in Table 1. As shown in Table 1, intratracheal instillation of AgNPs significantly decreased LVFS from $(41.35 \pm 1.10)$ in control to $(37.65$ $\pm 1.92)$ in AgNPs group $(P<0.05)$ and EF from $(77.46$ $\pm 0.98)$ to $(73.29 \pm 2.54)$ in $\operatorname{AgNPs}(P<0.05)$. There was no significant difference in other echocardiography indicators, which include PA, IVS, LVIDd, LVIDs, SV, EDV, and $\mathrm{ESV}$, among the four groups. However, when the cardiac output $(\mathrm{CO})$ was calculated by multiplying stroke volume with heart rate, it showed that $\mathrm{CO}$ significantly decreased from $(235.34 \pm 22.16)$ in Control to $(192.09 \pm 26.49)$ in AgNPs groups $(P<0.05)$, suggesting that AgNPs impaired cardiac function. Although $\mathrm{CO}$ did not significantly increase, selenite treatment brought the levels of LVFS and EF back to the control level ( $P>0.05$ vs Control).
A

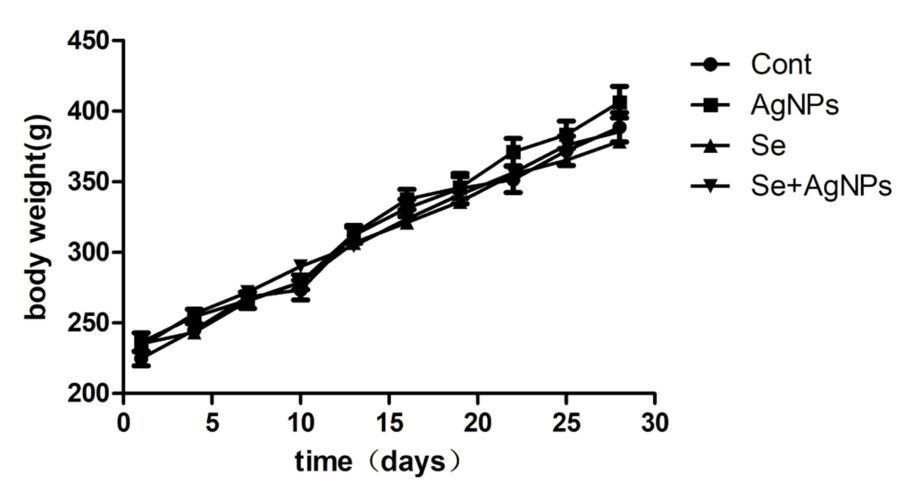

B

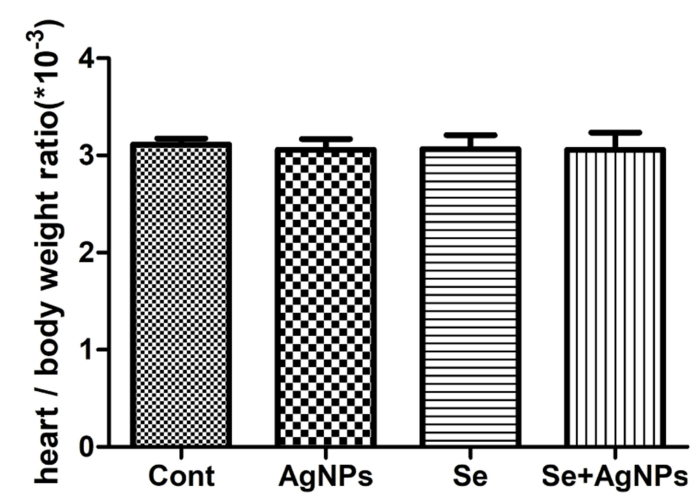

Figure I The assessment of body weight and the heart coefficient ratio. (A) Body weight of all four groups of rats was recorded twice a week, which increased in a similar trend. (B) The heart coefficient ratio was calculated and analyzed. Groups with no label on the top indicate no significant difference ( $P>0.05)$ when compared among the groups.

Notes: The data represent means $\pm S D$. $p<0.05$ were considered to be statistically significant.

Abbreviations: AgNPs, silver nanoparticles; Se, sodium selenite; SD, standard deviation. 
Table I Echocardiography Measurements in Rats Treated with or Without AgNPs and Selenite

\begin{tabular}{|l|l|l|l|l|}
\hline Parameters & Cont & AgNPs & Se & AgNPs+Se \\
\hline PA (mm) & $3.03 \pm 0.12$ & $3.00 \pm 0.06$ & $3.13 \pm 0.15$ & $3.10 \pm 0.17$ \\
IVS (mm) & $1.42 \pm 0.04$ & $1.45 \pm 0.05$ & $1.45 \pm 0.05$ & $1.43 \pm 0.05$ \\
LVIDd (mm) & $7.17 \pm 0.27$ & $7.13 \pm 0.37$ & $7.10 \pm 0.51$ & $7.17 \pm 0.31$ \\
LVIDs (mm) & $4.23 \pm 0.19$ & $4.45 \pm 0.31$ & $4.17 \pm 0.29$ & $4.13 \pm 0.32$ \\
LVFS (\%) & $41.35 \pm 1.10$ & $37.65 \pm 1.92^{*}$ & $41.52 \pm 1.68^{\#}$ & $42.39 \pm 2.33^{\#}$ \\
EF (\%) & $77.46 \pm 0.98$ & $73.29 \pm 2.54^{*}$ & $77.79 \pm 1.78^{\#}$ & $78.49 \pm 2.51^{\#}$ \\
CO (mL/min) & $235.34 \pm 22.16$ & $192.09 \pm 26.49 *$ & $222.56 \pm 37.04$ & $225.19 \pm 47.63$ \\
SV (mL) & $0.65 \pm 0.07$ & $0.60 \pm 0.08$ & $0.63 \pm 0.12$ & $0.65 \pm 0.07$ \\
EDV $(\mathrm{mL})$ & $0.84 \pm 0.09$ & $0.82 \pm 0.11$ & $0.81 \pm 0.15$ & $0.83 \pm 0.11$ \\
ESV $(\mathrm{mL})$ & $0.19 \pm 0.02$ & $0.22 \pm 0.04$ & $0.18 \pm 0.03$ & $0.18 \pm 0.04$ \\
\hline
\end{tabular}

Notes: The data represent means \pm SD. ${ }^{*} P<0.05$ vs control; ${ }^{\#} P<0.05$ vs AgNPs.

Abbreviations: Cont, control; AgNPs, silver nanoparticles; Se, selenium; PA, pulmonary artery internal diameter; IVS, interventricular septum thickness; LVIDd, the left ventricular internal dimension at the end of diastole; LVIDs, left ventricular internal dimension at the end of systole; LVFS, the left ventricular fractional shortening; EF, ejection fraction; CO, cardiac output; SV, stroke volume; EDV, the left ventricular volume at the end of diastole; ESV, the left ventricular volume at the end of systole.

Selenite administration alone did not affect any of the measured parameters.

In order to further clarify the causes of LVFS and EF changes, the invasive hemodynamic measurement was carried out. As shown in Table 2, significant decreases in HR, LVSP, $\mathrm{LVdP} / \mathrm{dt}_{\max }$ and $\mathrm{LVdP} / \mathrm{dt}_{\text {mix }}$, and an increase in LVEDP were observed in the AgNPs-treated group in comparison with the Control group. Treatment with selenite markedly increased LVSP, $\mathrm{LVdP} / \mathrm{dt}_{\text {max }}$ and $\mathrm{LVdP} / \mathrm{dt}_{\text {mix }}$, and reduced the LVEDP levels. Representative echocardiograms (M-mode images) and left ventricular pressure diagrams in each group are presented in Figure 2.

\section{Ultrastructural Changes in Heart Tissue}

Cardiac histopathological changes were observed by HE staining and were shown in Figure S1.

There was no significant difference in the histopathological outcomes of heart tissue between control and AgNPstreated groups. Therefore, transmission electron microscopy observation of ultrastructural changes in the left ventricular myocardium was performed and significant differences between the control and AgNPs-treated groups were observed (Figure 3). Sample from control animals demonstrated regular arrangement of sarcomeres with the myofibrils (F) nicely arranged in zones and bands and abundant mitochondria (M) in myocardiocytes. Striations were clearly visible among the well-organized myofilaments with M-lines intersecting the H-bands. The A-bands, I-bands, and Z-lines look clear. The electron microscopy of the Se-treated group showed in a normal way. Intratracheal instillation of AgNPs resulted in obvious fragmentation and lysis of the myofibrils and disarray of the sarcomere. The A-bands, Z-lines, $\mathrm{H}$-bands, and M-lines were all misarranged and damaged at various degrees. These abnormalities reverted to normal status with selenite treatment to AgNPs intratracheal instillation animals (AgNPs+Se group).

The ultrastructural changes of myocardial capillaries and their endothelial cells were examined by transmission electron microscopy and presented in Figure 4A. The capillary lumens were clearly visible and the nuclear of the endothelial cell is normal in both the Control and Se-treated groups. After AgNPs exposure, endothelial cell proliferation and

Table 2 Hemodynamics Measurements in Rats Treated with or Without AgNPs and Selenite

\begin{tabular}{|c|c|c|c|c|}
\hline Parameters & Cont & AgNPs & Se & AgNPs+Se \\
\hline HR (beats/min) & $437.83 \pm 22.16$ & $387.17 \pm 31.61 *$ & $429.67 \pm 4.23^{\#}$ & $4 \mid 2.00 \pm 4.00$ \\
\hline $\operatorname{LVSP}(\mathrm{mmHg})$ & $117.84 \pm 1.99$ & $93.27 \pm 8.56 *$ & $109.43 \pm 9.95^{\#}$ & $108.60 \pm 7.94^{\#}$ \\
\hline LVEDP (mmHg) & $15.95 \pm 2.84$ & $25.74 \pm 6.19 *$ & $15.07 \pm 3.27^{\#}$ & $14.67 \pm 2.65^{\#}$ \\
\hline $\mathrm{LVdP} / \mathrm{dt}_{\max }(\mathrm{mmHg} / \mathrm{s})$ & $3015.49 \pm 61.16$ & $1659.60 \pm 355.87^{*}$ & $2697.76 \pm 297.27^{\#}$ & $2649.76 \pm 314.64^{\#}$ \\
\hline $\mathrm{LVdP} / \mathrm{dt}_{\min }(\mathrm{mmHg} / \mathrm{s})$ & $2555.28 \pm 31.29$ & $1489.58 \pm 337.19 *$ & $2331.41 \pm 175.54^{\#}$ & $2373.38 \pm 345.32^{\#}$ \\
\hline
\end{tabular}

Notes: The data represent means \pm SD. $* P<0.05$ vs control; ${ }^{\#} P<0.05$ vs AgNPs

Abbreviations: Cont, control; AgNPs, silver nanoparticles; Se, selenium; HR, heart rate; LVSP, left ventricular systolic pressure; LVEDP, left ventricular end-diastolic pressure; $L V d P / d t_{\max }$, maximal rate of left ventricular pressure rise; $L V d P / d t_{\min }$, maximal rate of pressure decrease during relaxation. 
A

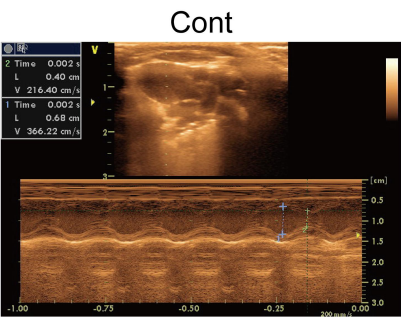

B

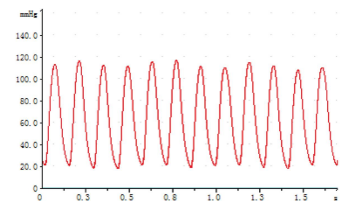

LVSP: $116.87 \mathrm{mmHg}$ LVEDP: $17.73 \mathrm{mmHg}$
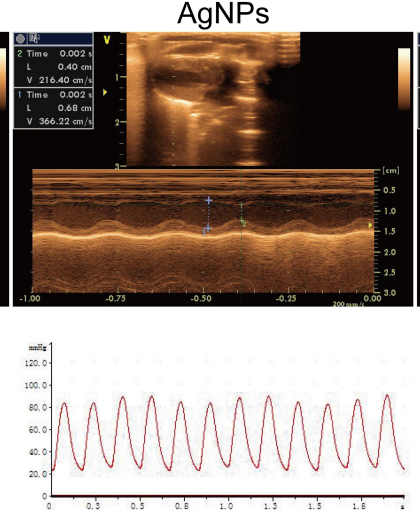

LVSP: $90.65 \mathrm{mmHg}$ LVEDP: $22.49 \mathrm{mmHg}$
Se
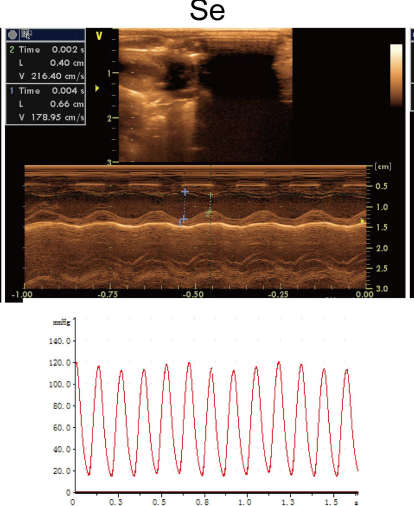

LVSP:120.46mmHg LVEDP: $14.43 \mathrm{mmHg}$
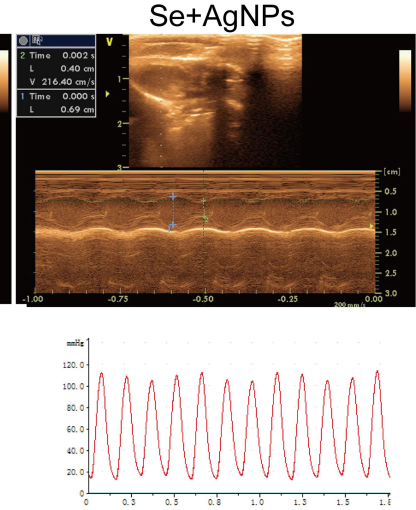

LVSP: $114.37 \mathrm{mmHg}$ LVEDP: $12.60 \mathrm{mmHg}$

Figure 2 Assessment of cardiac function by echocardiography and invasive hemodynamics. (A) Representative M-mode echocardiograms images were captured in each rat. (B) Representative left ventricular pressure diagrams were recorded in each group.

Abbreviations: Cont, control; AgNPs, silver nanoparticles; Se, selenium; LVSP, left ventricular systolic pressure; LVEDP, left ventricular diastolic pressure.
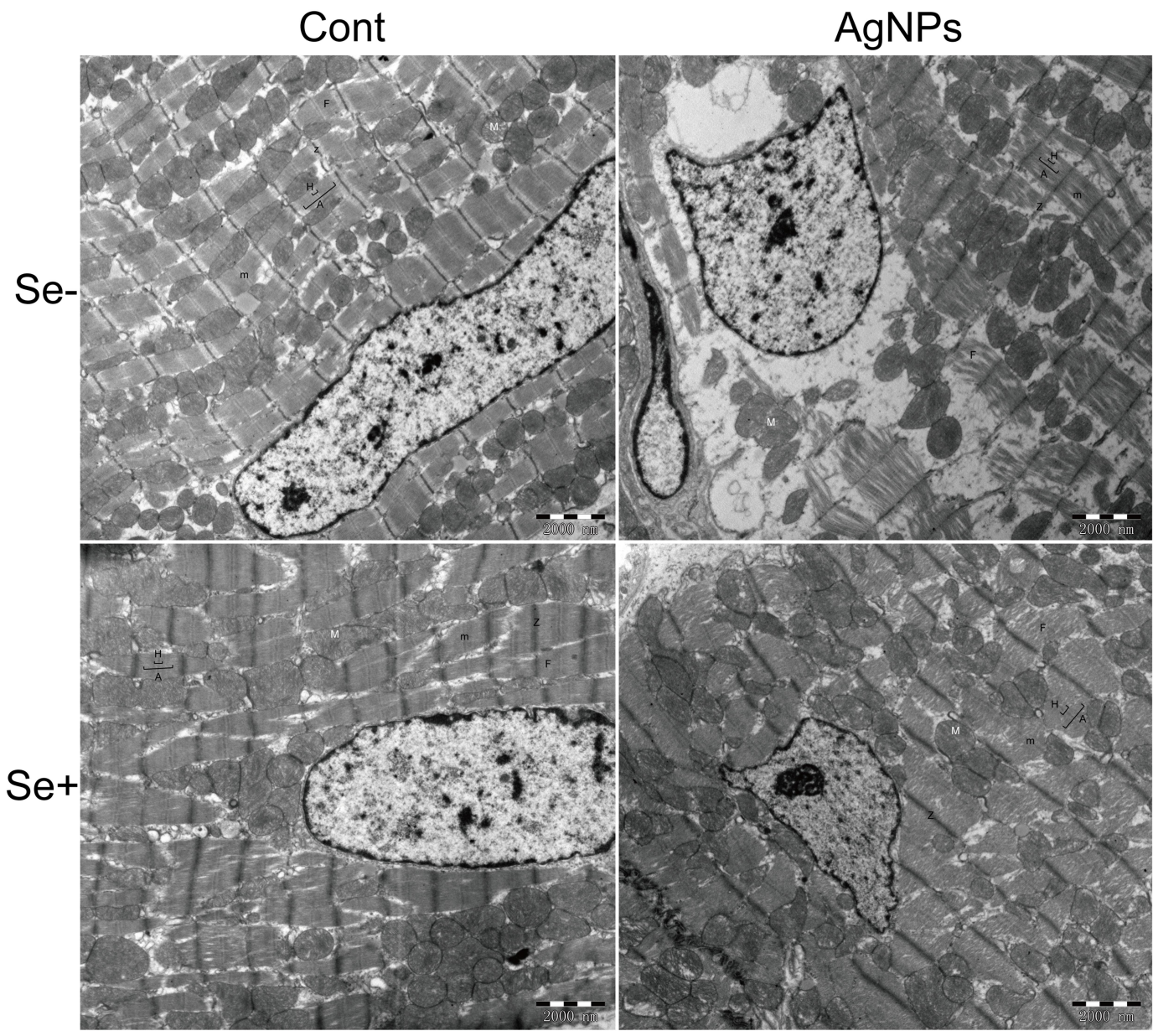

Figure 3 Ultrastructural changes of heart tissue observed by 10,000×TEM. Sarcomere and myofilament were observed. After exposure of AgNPs, fragmentation and lysis of the myofibrils and disarray of the sarcomere were found.

Abbreviations: Cont, control; AgNPs, silver nanoparticles; Se, selenium; H, H-bands; A, A-bands; m, M-lines; Z, Z-lines; F, myofibrils; M, mitochondria. 
A
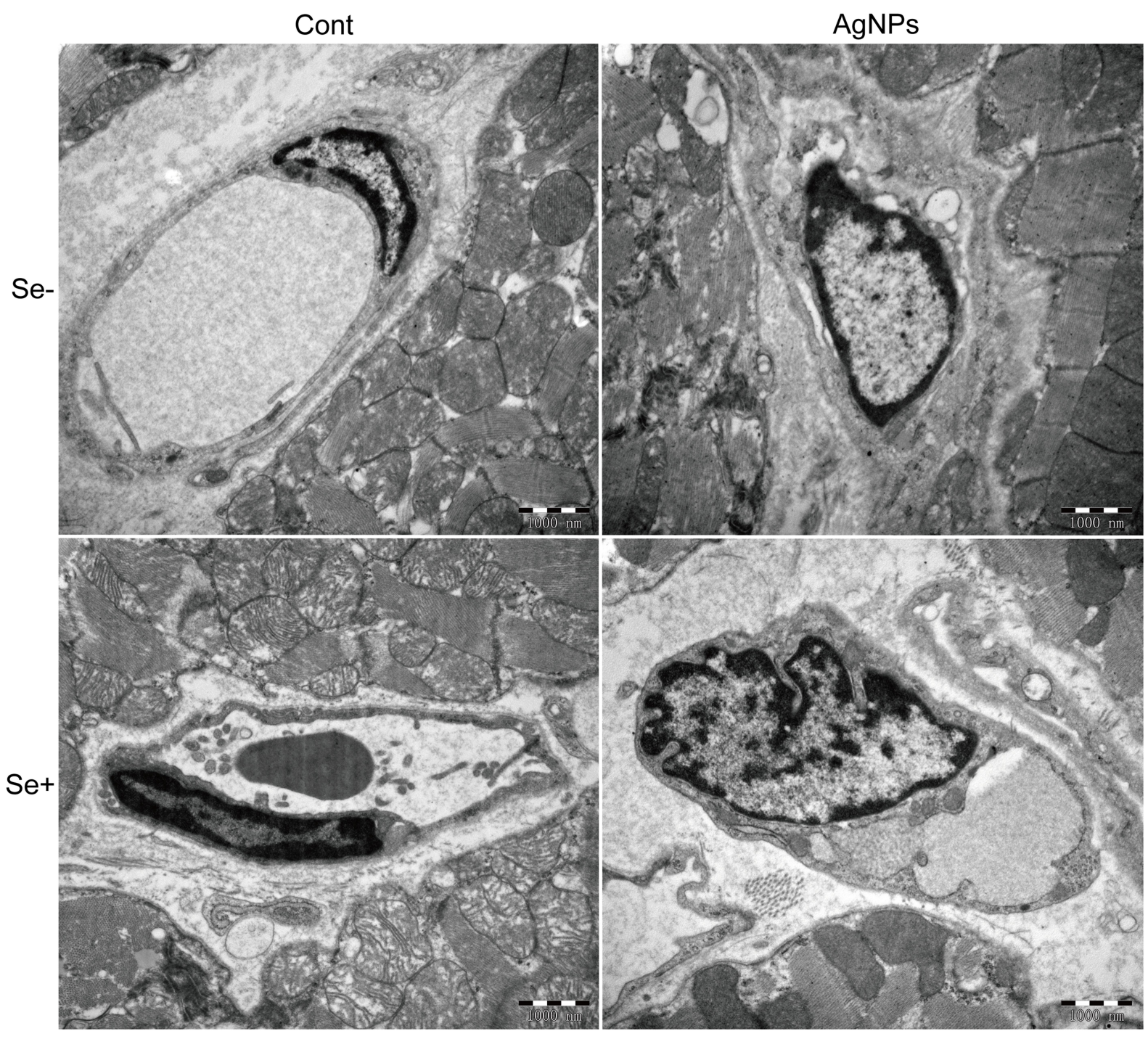

B
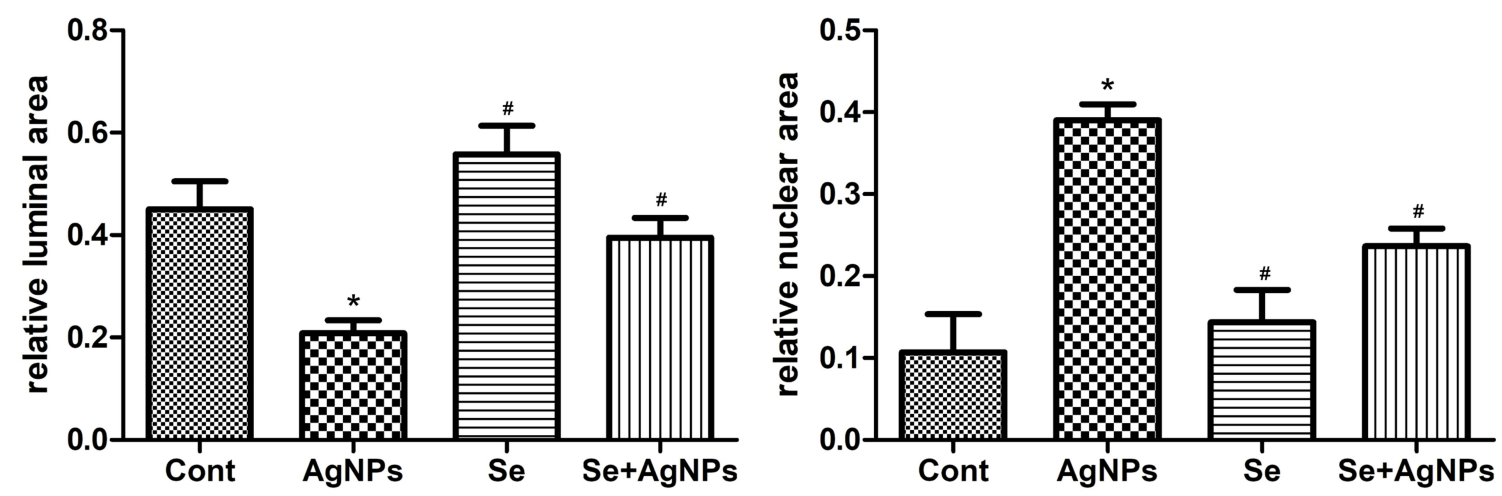

Figure 4 Ultrastructural changes of myocardial capillaries observed by 20,000×TEM. (A) Ultrastructural changes in myocardial capillaries and their endothelial cells. (B) The summarized bar graph showing the relative luminal area and the relative nuclear area.

Notes: The Data were collected from ten independent images and presented as means $\pm S D$. $p<0.05$ were considered to be statistically significant. * $p<0.05$ vs control; $\# p<0.05$ vs AgNPs.

Abbreviations: Cont, control; AgNPs, silver nanoparticles; Se, selenium; SD, standard deviation. 
nuclear chromatin margination were observed. The nucleus bulged into the lumen of capillary leading to stenosis. In the AgNPs+Se cotreated animals, the AgNPs-induced changes in the capillaries improved. The ratio of vascular lumen area to the vascular area and the ratio of nuclear area to the vascular area were measured using the Image $\mathrm{J}$ program and given in Figure 4B. The relative luminal area was 0.45 \pm 0.17 and $0.55 \pm 0.18$ in control and Se-treated groups, respectively. The relative nuclear area was $0.11 \pm 0.13$ and $0.14 \pm 0.11$ in control and Se-treated groups, respectively. Intratracheal instillation of AgNPs decreased the relative luminal area to $0.21 \pm 0.08$ ( $P<0.05$ vs Control) and increased the relative nuclear area to $0.39 \pm 0.05$ ( $P<0.05$ vs Control). Selenite treatment in AgNPs-exposed animals significantly increased the relative luminal area up to $0.39 \pm 0.12(P<0.05$ vs AgNPs) and decreased the relative nuclear area to 0.23 $\pm 0.06(P<0.05$ vs AgNPs).

\section{Ultrastructural Changes of Mitochondrial Morphology and the Mitophagy}

Given the critical role of mitochondria in myocardial energy supply, the morphology changes of mitochondria were evaluated by transmission electron microscopy. As shown in Figure 5, the mitochondrial cristae were well arranged and abundant and mitochondrial membranes were intact in Control and Se groups. After AgNPs exposure, the mitochondrial membrane partially disappeared and the cristae became blurry. Silver nanoparticles (green arrows in Figure 5C) were found within the myocardiocyte mitochondria. In AgNPs+Se animals, the mitochondrial cristae returned to normal and AgNPs accumulation was observed within the mitochondria (green arrow in Figure 5D) and outside of the mitochondria (yellow arrows in Figure 5D). Strikingly, mitochondrial autophagy (mitophagy) was observed both in AgNPs and AgNPs+Se cotreated groups (red arrows in Figure 5E and $F$ ).

\section{Effects of AgNPs and Selenite on Mitochondrial Fission and Autophagy Markers}

In the present study, we measured the mitochondrial fission and mitophagy markers from the mitochondrial fraction of the heart tissue samples. As shown in Figure 6, the protein levels of Drp1, p-Drp1, and Fis1 were significantly increased
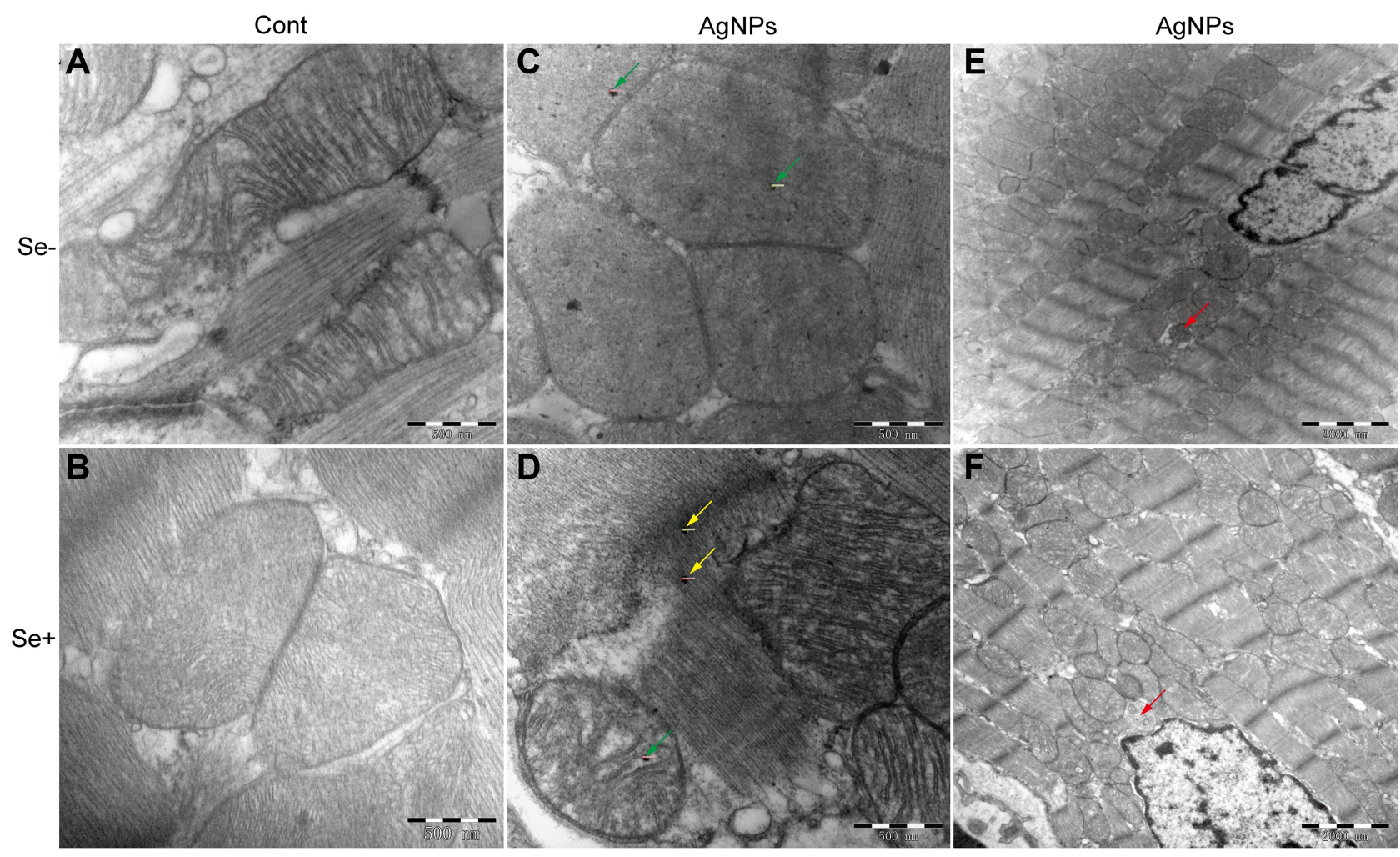

Figure 5 Ultrastructural changes of mitochondrial morphology and the mitophagy. (A-D) representative images showing mitochondrial morphology from (A) control, (B) Se-treated, (C) AgNPs-treated and (D) Se+AgNPs cotreated group; Green arrows denote silver nanoparticles within the mitochondria and yellow for outside. (E and $\mathbf{F}$ ) representative images showing mitophagy in (E) AgNPs-treated and (F) Se+AgNPs cotreated group. Red arrows denote mitophagy.

Abbreviations: Cont, control; AgNPs, silver nanoparticles; Se, selenium. 
A

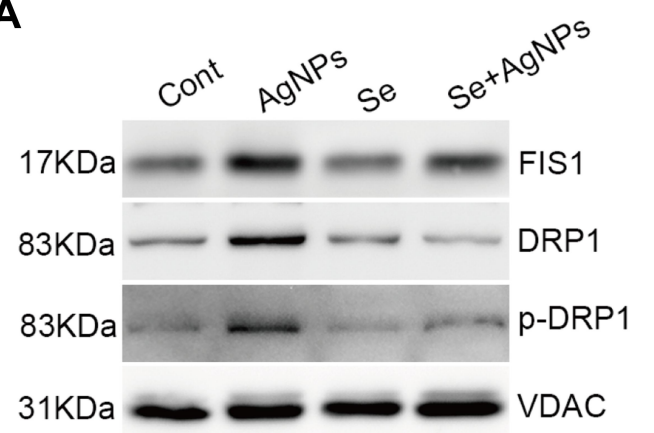

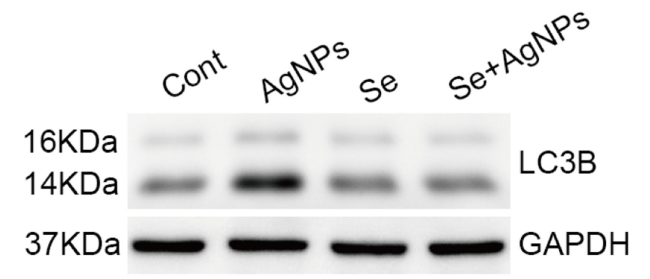

Cont $A g^{N P s}$ se $s e^{x+g^{N P s}}$

C3B

APDH

B
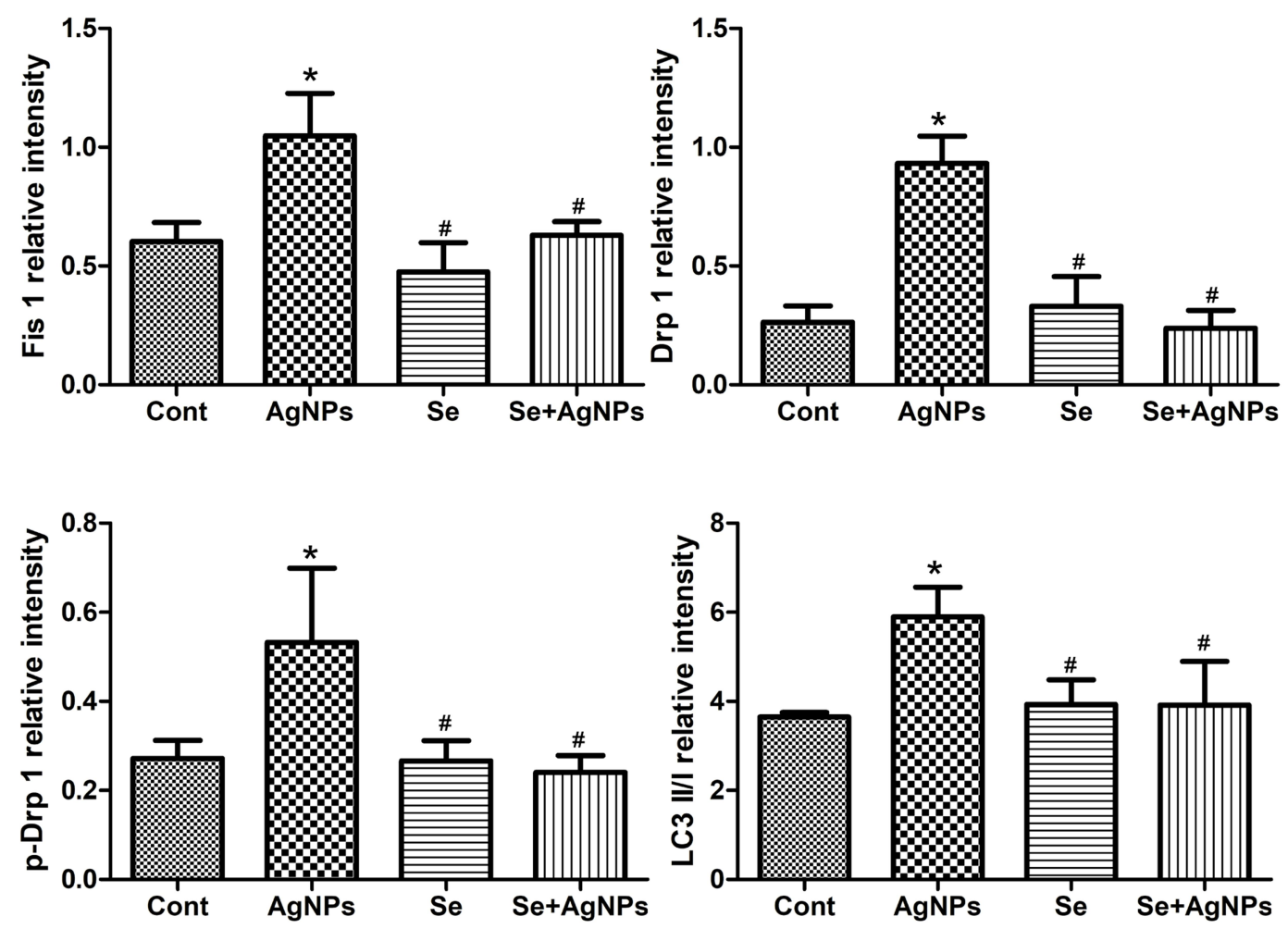

Figure 6 Western blotting of mitochondrial fission and autophagy markers. (A) The representative Western blot showing Drpl, p-DrpI, Fis I and LC3B levels. VDAC and GAPDH served as the internal loading control for mitochondrial and whole protein, respectively. (B) The bar graph showing semiquantitative band intensity ratio from DrpI, P-Drpl, FisI to LC3B.

Notes: The data represent means \pm SD. $* p<0.05$ vs control; \#p<0.05 vs AgNPs.

Abbreviations: Cont, control; AgNPs, silver nanoparticles; Se, selenium; SD, standard deviation.

in the AgNPs-treated group compared with the control group (Drp1, $0.936 \pm 0.205$ vs $0.259 \pm 0.121$; p-Drp1, $0.532 \pm 0.166$ vs $0.027 \pm 0.04$; Fis $1,1.049 \pm 0.178$ vs.0.602 \pm 0.080$)$. Treatment with selenite for 7 days significantly reduced these protein levels to the control levels. Further selenium treatment suppressed AgNPs-activated autophagy. The conversion of LC3 I to LC3 II is an important marker of autophagy. It was shown that the levels of LC3 II band intensities significantly increased from $(3.938 \pm 0.428)$ in Control to $(6.414 \pm 0.547)$ in the AgNPs-treated group and selenite reverted the AgNPsinduced elevation back to control levels.

\section{Discussion}

The present study has demonstrated that AgNPs caused ultrastructural changes in the heart tissue, which include damages to the mitochondria and capillaries, and increased the protein markers of mitochondrial fission and autophagy. These structural alterations ultimately resulted in cardiac functional 
performance as detected by hemodynamic and echocardiography. Treatment with selenium effectively alleviated AgNPsinduced functional and ultrastructural deterioration in the heart and prevented the elevations of mitochondrial fission and autophagy proteins.

Numerous research studies have shown that the AgNPs toxicity is mostly particle size-dependent both in vivo and in vitro. ${ }^{31-33}$ In our study, the size of silver nanoparticles was $20 \mathrm{~nm}$ and the dose we used is consistent with those studying cardiotoxicity of AgNPs. ${ }^{15,34}$ Although neither AgNPs nor selenite affect the body weight and heart/body weight ratio, our data demonstrated that exposure of AgNPs leads to cardiac systolic dysfunction as reflected by the decreased levels of $\mathrm{CO}$, EF, and LVFS. Since CO, EF, and LVFS are the most important indicators of systolic function, hemodynamic experiments were carried out to further confirm the effect of AgNPs on cardiac function. The results showed that intratracheal instillation of AgNPs significantly decreased the level of HR, LVSP, $\mathrm{LVdP} / \mathrm{dt}_{\max }$ and $\mathrm{LVdP} / \mathrm{dt}_{\min }$, and increased the level of LVEDP. LVSP is an indicator of ejection efficiency during myocardial contraction and its decrease indicates reduced cardiac muscle contraction of the left ventricle. LVEDP is the left ventricular end of diastolic pressure and its elevation indicates the pressure in the left ventricular at the end of diastole increased, which reduces the ventricle blood filling. ${ }^{35}$ $\mathrm{LVdP} / \mathrm{dt}_{\max }$, a sensitive and reliable index of cardiac contractility, and $\mathrm{LVdP} / \mathrm{dt}_{\mathrm{min}}$, reflecting the effects on myocardial relaxation, are closely related to the systolic and diastolic functions of the myocardium. ${ }^{36,37}$ In other words, exposure of AgNPs caused cardiovascular damage ranging from decreased myocardial contractility to subsided diastolic dysfunction. Our results are consistent with those of a previous study showing that AgNPs causes a decrease in myocardial contractility assessed by echocardiography. ${ }^{12}$ Sodium selenium treatment significantly prevented AgNPs-induced cardiac systolic dysfunction as evidenced by the improvement of EF, LVFS, LVSP and LVdP/dt $t_{\max }$, and improved left ventricular diastolic dysfunction as reflected by returning of LVEDP and $\mathrm{LVdP} / \mathrm{dt}_{\text {min }}$ back to normal. Taking together, our results suggests that AgNPs causes dysfunctions of both myocardial contraction and relaxation and selenite treatment attenuated or prevented these AgNPs-induced cardiac dysfunctions in rats.

AgNPs exposure affects not only cardiac function but also cardiac ultrastructure.

Although hypertrophy, edema, vasodilation, hyperemia, and rupture of myocardial fibers were not observed in the AgNPs group by HE staining, the ultrastructure of myocardial cells was altered as observed by transmission electron microscopic examination. The results showed that exposure to AgNPs could cause disarray of sarcomere, fragmentation of myofibrillary fibers, and stenosis of the capillary lumen. These structural impairments may explain why cardiac hemodynamic parameters were affected and cardiac functions were reduced in AgNPs-exposed animals. Although there have been studies reported that selenium alleviates heavy metal and silver induced toxicity in cells and tissues, ${ }^{23,38-40}$ there has been no study examining the detoxifying effect of sodium selenite on AgNPsinduced toxicity in cardiac tissue. The results from this study clearly demonstrated that treatment with selenite reduced the disarray of sarcomere and fragmentation of myofibrils, and improved capillary patency in AgNPsexposed rats, suggesting that selenium supplementation is capable of correcting AgNPs toxicity in the heart.

Mitochondria are the most important organelles for energy metabolism. The heart is the most metabolically active organ, which is highly dependent on the energy production in mitochondria to provides a large amount of adenosine triphosphate (ATP) to maintain its continuous contractile activity. So cells residing within the heart tissue are highly metabolically active and also more sensitive to mitochondrial damage. ${ }^{17,41}$ The mitochondrial inner membrane forms cristae, which contains the respiratory complexes, ATP synthase, ADP/ATP carrier, and phosphate carrier. ${ }^{42}$ Healthy mitochondria and cristae are very important for the capacity of ATP generation, especially for the heart. In our study, the morphology of mitochondria was observed by transmission electron microscopic examination. After AgNPs exposure, the mitochondrial membrane partially was disappeared in some part and the cristae became blurred. Silver nanoparticles were founded within the mitochondria of the myocardiocytes. Recently, it has been reported that AgNPs compromised hepatic mitochondrial activity and mitochondrial respiratory complex activities in Sprague-Dawley rats. ${ }^{43}$ Unfortunately, we were unable to replicate these results in heart tissue due to the limited amount of available tissue samples. Although there were silver nanoparticles in and out of the mitochondria, the mitochondria cristae return to normal in the Se-treated group. Therefore, selenite is capable of protecting the mitochondria against AgNPs-induced damage.

In general, the mitochondria are highly dynamic in an equilibrium of fission and fusion, which can not only regulate the quantity, morphology, and subcellular distribution of mitochondria but also maintain the functions of mitochondria. Mitochondrial fission is a process of 
packaging degenerated mitochondria and metabolic wastes and eliminating or recycling them through mitochondrial autophagy. ${ }^{20}$ Excessive fission is often associated with mitochondrial dysfunction as this dynamic state is dominantly elevated in stress and cell death processes. $^{44}$ Our data showed that AgNPs significantly increased mitochondrial fission protein levels of Drp1, p-Drp1 (Ser616), and Fis1, suggesting that AgNPs exposure activated mitochondrial fission. Selenite treatment almost completely blocked the AgNPs-induced increases of Drp1, p-Drp1, and Fis1. Autophagy is an important process in cell homeostasis and clearance of redundant or damaged organelles. ${ }^{45}$ Since fission mitochondria need to be cleared through autophagy, we measured one of the major autophagy marker LC-3. ${ }^{19}$ The results showed that the ratio of LC3-II to LC3-I increased significantly after AgNPs exposure and selenite decreased the conversion of LC3-I to LC3-II under AgNPs exposure. It has been shown that the conversion of LC-3I to LC-3II plays a critical role in the process of engulfing defective mitochondria into autophagosomes and then delivering them to the lysosomes for degradation. ${ }^{46}$ Our results suggest that AgNPs activates mitochondrial fission and autophagy processes and selenite successfully block these processes. Sodium selenite has been shown to prevent mitochondrial dynamic imbalance and improve mitochondrial function in neuronal cells by our group previously. ${ }^{47,48}$ Results obtained from transmission electron microscopy further supported this conclusion by verifying the existence of double-layered, mitochondrion-containing autophagy bodies in myocardiocytes of AgNPs-exposed animals.

\section{Conclusion}

Our results showed that the sodium selenite is capable of alleviating AgNPs-induced myocardiocyte damage. Intratracheal instillation of AgNPs caused decreasing cardiac contraction, diastolic dysfunction, fragmentation, and lysis of the myofibrils, the formation of stenosis in the capillary, damaging the mitochondria membrane and cristae. AgNPs activated mitochondrial fission and autophagy. Treatment with sodium selenite to the animals prevented the AgNPs-caused functional and ultrastructural alterations, stabilized mitochondrial dynamic balance, and blocked mitochondrial autophagy.

\section{Acknowledgments}

Our research was supported by the Natural Science Foundation of China under Grant (81660530) to WM and partially supported by US NSF-Excellence in Research under Grant (1832134) to JB. The authors greatly appreciate Dr. Tracie Locklear for proof-read this manuscript.

\section{Disclosure}

The authors report no conflicts of interest in this work.

\section{References}

1. Lin CX, Yang SY, Gu JL, et al. The acute toxic effects of silver nanoparticles on myocardial transmembrane potential, INa and IK1 channels and heart rhythm in mice. Nanotoxicology. 2017;11 (6):827-837.

2. Hendren CO, Mesnard X, Droge J, et al. Estimating production data for five engineered nanomaterials as a basis for exposure assessment. Environ Sci Technol. 2011;45(7):2562-2569.

3. Yu SJ, Yin YG, Liu JF. Silver nanoparticles in the environment. Environ Sci Process Impacts. 2013;15(1):78-92. doi:10.1039/ C2EM30595J

4. Bachler G, Goetz NV, Hungerbühler K. A physiologically based pharmacokinetic model for ionic silver and silver nanoparticles. Int J Nanomedicine. 2013;8:3365-3382.

5. Stebounova LV, Adamcakova-Dodd A, Kim JS, et al. Nanosilver induces minimal lung toxicity or inflammation in a subacute murine inhalation model. Part Fibre Toxicol. 2011;8(1):5.

6. Driscoll KE, Costa DL, Hatch G, et al. Intratracheal instillation as an exposure technique for the evaluation of respiratory tract toxicity: uses and limitations. Toxicol Sci. 2000;55(1):24-35.

7. Johnston HJ, Hutchison G, Christensen FM, et al. A review the in vivo and in vitro toxicity of silver and gold particulates: particle attributes and biologimechanisms responsible for the observed toxicity. Crit Rev Toxicol. 2010;40(4):328-346.

8. Takenaka S, Karg E, Roth C, et al. Pulmonary and systemic distribution of inhaled ultrafine silver particles in rats. Environ Health Perspect. 2001;109(Suppl 4):547-551.

9. Korani M, Rezayat SM, Bidgoli SA. Sub-chronic dermal toxicity of silver nanoparticles in guinea pig: special emphasis to heart, bone and kidney toxicities. Iran J Pharm Res. 2013;12(3):511-519.

10. Manuel RA, Martinez-Cuevas PP, Rosas-Hernandez H, et al. Evaluation of vascular tone and cardiac contractility in response to silver nanoparticles, using Langendorff rat heart preparation. Nanomedicine. 2017;13(4):1507-1518.

11. Ramirez-Lee MA, Aguirre-Bañuelos P, Martinez-Cuevas PP, et al. Evaluation of cardiovascular responses to silver nanoparticles (AgNPs) in spontaneously hypertensive rats. Nanomedicine. 2018;14(2):385-395.

12. Raieszadeh H, Noaman V, Yadegari M. Echocardiographic assessment of cardiac structural and functional indices in broiler chickens treated with silver nanoparticles. Sci World J. 2013;27:931432.

13. Taju G, Majeed SA, Nambi KSN, et al. In vitro assay for the toxicity of silver nanoparticles using heart and gill cell lines of Catla catla and gill cell line of Labeo rohita. Comp Biochem Physiol C Toxicol Pharmacol. 2014;161:41-52.

14. Elle RE, Gaillet S, Romain JVC, et al. Dietary exposure to silver nanoparticles in Sprague-Dawley rats: effects on oxidative stress and inflammation. Food Chem Toxicol. 2013;60:297-301.

15. Holland NA, Thompson LC, Vidanapathirana AK, et al. Intratracheal instillation of silver nanoparticles exacerbates cardiac ischemia/reperfusion injury in male sprague-dawley rats. The Scholarship. 2014;13:48.

16. Duan J, Yu Y, Li Y, et al. Low-dose exposure of silica nanoparticles induces cardiac dysfunction via neutrophil-mediated inflammation and cardiac contraction in zebrafish embryos. Nanotoxicology. 2016;10(5):575-585. 
17. Maurer LL, Meyer JN. A systematic review of evidence for silver nanoparticle-induced mitochondrial toxicity. Environ Sci Nano. 2016;3(2):311-322.

18. Neely JR, Morgan HE. Relationship between carbohydrate and lipid metabolism and the energy balance of heart muscle. Annu Rev Physiol. 1974;36:413-459.

19. Jahani-Asl A, Cheung EC, Neuspiel M, et al. Mitofusin 2 protects cerebellar granule neurons against injury-induced cell death. $\mathrm{J}$ Biol Chem. 2007;282(33):23788-23798.

20. Wu S, Zhou F, Zhang Z, et al. Mitochondrial oxidative stress causes mitochondrial Fragmentation via differential modulation of mitochondrial fission-fusion proteins. FEBS J. 2011;278(6):941-954.

21. Stoll S, Leimena C, Qiu HY. Mitochondria and Heart Disease. Mitochondria Disease. 2nd ed. Intechopen USA; 2018:31-59.

22. Li HN, Zimmerman M, Milledge GZ, et al. Water-soluble coenzyme Q10 reduces rotenone-induced mitochondrial fission. Neurochem Res. 2017;42(4):1096-1103.

23. Ma W, Jing L, Valladares A, et al. Silver nanoparticle exposure induced mitochondrial stress, caspase-3 activation and cell death: amelioration by sodium selenite. Int $J$ Biol Sci. 2015;11 (8):860-867.

24. Ma W, He S, Ma H, et al. Silver nanoparticle exposure causes pulmonary structural damage and mitochondrial dynamic imbalance in the rat: protective effects of sodium selenite. Int J Nanomedicine. 2020;15:633-645.

25. IKemoto T, Kunito T, Tanaka H, et al. Detoxification mechanism of heavy metals in marine mammals and seabirds: interaction of selenium with mercury, silver, copper, zinc, and cadmium in liver. Arch Environ Contam Toxicol. 2004;47(3):402-413. doi:10.1007/s00244004-3188-9

26. Islam F, Zia S, Sayeed I, et al. Selenium-induced alteration of lipids, lipid peroxidation, and thiol group in circadian rhythm centers of rat. Biol Trace Elem Res. 2002;90(1-3):203-214. doi:10.1385/ BTER:90:1-3:203

27. Venardos K, Harrison G, Headrick J, et al. Effects of dietary selenium on glutathione peroxidase and thioredoxin reductase activity and recovery from cardiac ischemia-reperfusion. J Trace Elem Med Biol. 2004;18(1):81-88.

28. Panee J, Liu W, Nakamura K, et al. The responses of HT22 cells to the blockade of mitochondrial complexes and potential protective effect of selenium supplementation. Int $J$ Biol Sci. 2007;3 (5):335-341. doi:10.7150/ijbs.3.335

29. Srivastava M, Singh S, Self WT. Exposure to silver nanoparticles inhibits selenoprotein synthesis and the activity of thioredoxin reductase. Environ Health Perspect. 2012;120(1):56-61. doi:10.1289/ehp.1103928

30. Shipelin VA, Kudan PV, Zgoda VG, et al. Effect of silver nanoparticles on protein composition of rat liver microsomal fraction. Bull Exp Bio Med. 2018;166(1):80-85. doi:10.1007/s10517-018-4293-5

31. Siddiqi KS, Husen A, Rao RAK. A review on biosynthesis of silver nanoparticles and their biocidal properties. J Nanobiotechnology. 2018;16(1):14
32. Braakhuis HM, Cassee FR, Fokkens PH, et al. Identification of the appropriate dose metric for pulmonary inflammation of silver nanoparticles in an inhalation toxicity study. Nanotoxicology. 2016;10 (1):63-73.

33. Recordati C, Maglie MD, Bianchessi S, et al. Tissue distribution and acute toxicity of silver after single intravenous administration in mice: nano-specific and size-dependent effects. Part Fibre Toxicol. 2016;3:12.

34. Holland NA, Becak DP, Shannahan JH, et al. Cardiac ischemia reperfusion injury following instillation of $20 \mathrm{~nm}$ citrate-capped nanosilver. J Nanomed Nanotechnol. 2015;6(Suppl 6):006.

35. Zhou R, Xu Q, Zheng P, et al. Cardioprotective effect of fluvastatin on isoproterenol-induced myocardial infarction in rat. Eur J Pharmacol. 2008;586(1-3):244-250.

36. Abd-El-Aziz TA. Noninvasive prediction of left ventricular end-diastolic pressure in patients with coronary artery disease and preserved deduction fraction. Can J Cardiol. 2012;28(1):80-86.

37. Pugsley MK, Guth B, Chiang AY, et al. An evaluation of the utility of $\mathrm{LVdP} / \mathrm{dt} 40$, QA interval, $\mathrm{LVdP} / \mathrm{dt}_{\min }$ and Tau as indicators of drug-induced changes in contractility and lusitropy in dogs. J Pharmacol Toxicol Methods. 2017;85:1-21.

38. Ansar S, Alshehri SM, Abudawood M, et al. Antioxidant and hepatoprotective role of selenium against silver nanoparticles. Int J Nanomedicine. 2017;12:7789-7797. doi:10.2147/IJN.S136748

39. Ansar S, Abudawood M, Hamed SS, et al. Sodium selenite protects against silver nanoparticle-induced testicular toxicity and inflammation. Bio Trace Elem Res. 2017;175(1):161-168.

40. Abarikwu SO, Adebayo OL, Otuechere CA, et al. Selenium and rutin alone or in combination do not have stronger protective effects than their separate effects against cadmium-induced renal damage. Pharm Biol. 2016;54(5):896-904.

41. Neustadt J, Pieczenik SR. Medication-induced mitochondrial damage and disease. Mol Nutr Food Res. 2008;52(7):780-788.

42. Ruprecht JJ, Kunji ER. Structural changes in the transport cycle of the mitochondrial ADP/ATP carrier. Curr Opin Struct Bio. 2019;57:135-144.

43. Teodoro JS, Sliva R, Varela AT, et al. Low-dose, subchronic exposure to silver nanoparticles causes mitochondrial alterations in Sprague-Dawley rats. Nanomedicine (Lond). 2016;11(11):1359-1375.

44. Zemirli N, Morel E, Molino D. Mitochondrial dynamics in basal and stressful conditions. Int J Mol Sci. 2018;19(2):564.

45. Levine B. Eating oneself and uninvited guests: autophagy-related pathways in cellular defense. Cell. 2005;120(2):159-162.

46. Baumann K. Autophagy: mitophagy receptors unravelled. Nat Rev Mol Cell Biol. 2015;16(10):580.

47. Kumari S, Mehta SL, Li PA. Glutamate induces mitochondrial dynamic imbalance and autophagy activation: preventive effects of selenium. PLoS One. 2012;7(6):e39382.

48. Mendelev N, Mehta SL, Idris H, et al. Selenite stimulates mitochondrial biogenesis signaling and enhances mitochondrial functional performance in murine hippocampal neuronal cells. PLoS One. 2012;7(10):e47910.

\section{Publish your work in this journal}

The International Journal of Nanomedicine is an international, peerreviewed journal focusing on the application of nanotechnology in diagnostics, therapeutics, and drug delivery systems throughout the biomedical field. This journal is indexed on PubMed Central, MedLine, CAS, SciSearch ${ }^{\mathbb{R}}$, Current Contents ${ }^{\mathbb{R}} /$ Clinical Medicine, $^{2}$
Journal Citation Reports/Science Edition, EMBase, Scopus and the Elsevier Bibliographic databases. The manuscript management system is completely online and includes a very quick and fair peer-review system, which is all easy to use. Visit http://www.dovepress.com/ testimonials.php to read real quotes from published authors. 